\title{
Quasi-periodic changes of three dimensional solar anisotropy of galactic cosmic rays for 1965-2014
}

\author{
R. Modzelewska ${ }^{1}$ \\ Siedlce University, Faculty of Sciences, \\ Konarski str. 208110 Siedlce, Poland \\ E-mail:renatam@uph.edu.pl
}

\section{M.V. Alania}

Siedlce University, Faculty of Sciences, Siedlce, Poland

Tbilisi State University, Insitute of Geophysics, Tbilisi, Georgia

E-mail: alania@uph.edu.pl

\begin{abstract}
We study features of the three dimensional (3D) solar anisotropy of galactic cosmic rays (GCR) for 19652014 (almost 5 solar cycles 20-24). We analyze the 27-day variations of the two dimensional (2D) GCR anisotropy in the ecliptic plane, and the north-south anisotropy normal to the ecliptic plane. We study the dependence of the 27-day variation of the 3D GCR anisotropy on the solar cycle and solar magnetic cycle. We demonstrate that the 27-day variations of the GCR intensity and anisotropy can be used as an important tool to study solar wind, solar activity and heliosphere.

We show that behavior of the 27 -variation of the $3 \mathrm{D}$ anisotropy verifies an existence of a stable longlived active heliolongitudes on the sun. This finding illustrates usefulness of the 27-day variation of the GCR anisotropy as a unique proxy to study solar wind, solar activity and heliosphere. We distinguish a tendency of the 22-year changes of the amplitudes of the 27-day variation of the 2D anisotropy connected with the solar magnetic cycle. We demonstrate that the amplitudes of the 27-day variation of the north-south component of the anisotropy vary upon the 11 year solar cycle, however, a dependence of the solar magnetic polarity hardly can be recognized. We show that the 27-day recurrences of the GG index and At component are in a high positive correlation, and both are highly correlated with By component of the heliospheric magnetic field.
\end{abstract}

36th International Cosmic Ray Conference -ICRC2019-

July 24th - August 1st, 2019

Madison, WI, U.S.A.

\footnotetext{
${ }^{1}$ Speaker

(C) Copyright owned by the author(s) under the terms of the Creative Commons

Attribution-NonCommercial-NoDerivatives 4.0 International License (CC BY-NC-ND 4.0).
} 


\section{Introduction}

Flux of galactic cosmic ray (GCR) measured at Earth consists of the isotropic and anisotropic parts. An isotropic part contains the various quasi-periodic changes with different time scales (from hours to several years), see e.g. [1-3 and references therein] and 3D spatial density gradients [4]. An anisotropic part generally is reflected in the solar diurnal variation (24 hours wave) assuming that while the Earth completes one rotation, a location of a source of anisotropic stream remains unchanged.

The mechanism of the solar diurnal anisotropy was explained by Ahluwalia and Dessler [5], and a little later, by Krymsky [6] and Parker [7], independently, based on the anisotropic diffusion-convection theory of GCR propagation in the heliosphere. Chen and Bieber [8] have shed light on this problem assuming that 3D GCR anisotropy is a combination of the 2D solar ecliptic and the north-south anisotropies. 2D solar ecliptic anisotropy results in the daily variation of the count rate of ground based detector (e.g. neutron monitor (NM) or muon telescope (MT)) that rotates with Earth; the north-south anisotropy is revealing a flow of GCR normal to the ecliptic plane. Swinson [9] proposed that the north-south anisotropy could have occurred due to the drift caused by positive heliocentric radial density gradient Gr of cosmic rays and the By component of the heliospheric magnetic field (HMF) (realized as the vector product, ByGr).

Among recent publications devoted to the GCR anisotropy one can mention, e.g., $[1,4,10-$ 12]. However, quasi-periodic changes of the GCR anisotropy connected with the solar rotation, (further in this paper called the 27-day variation) were studied rarely until the work of Alania et al. [13-14] for ecliptic plane anisotropy (2D case) and for the north-south component only for polar-located NMs by Owens et al. [15]. Swinson and coauthors [16-19] reported the significant correlation between the 27-day variation of the north-south anisotropy for MTs data and the tilt angle of the heliospheric neutral sheet. Alania et al. [13-14] and Gil et al. [20] analyzing the periods near the minima epochs of solar activity, demonstrated that the amplitude of the 27-day variation of the $2 \mathrm{D}$ anisotropy is less in the negative polarity period than in the positive polarity period of the HMF.

The main aim of this paper is two fold: (1) to investigate the 27-day variation of the 3D GCR solar anisotropy and its long-term changes during 5 solar cycles 20-24, and (2) to study the time lines of the 27-day variations of the north-south component of the GCR anisotropy and the 2D GCR anisotropy in the ecliptic plane. 


\section{27-day variation of the 2D GCR anisotropy in the ecliptic plane}

Unfortunately, up to present it is not available a complete precise method for simultaneous calculation of a radial Ar, azimuthal Af and latitudinal (north-south) At components of 3D GCR anisotropy based on the world wide network of NMs and MTs. The Ar and Af components can be calculated using the both GSM and harmonic analyses methods based on data of NMs with cut-off rigidities $<5 \mathrm{GV}$; in addition a latitudinal At (north-south) component is possible to calculate in scope of the arbitrary constant, only by GSM method. Additionally, At component can be estimated as a difference of two NMs located in regions of north and south poles [8], also, At can be estimated by directed MTs.

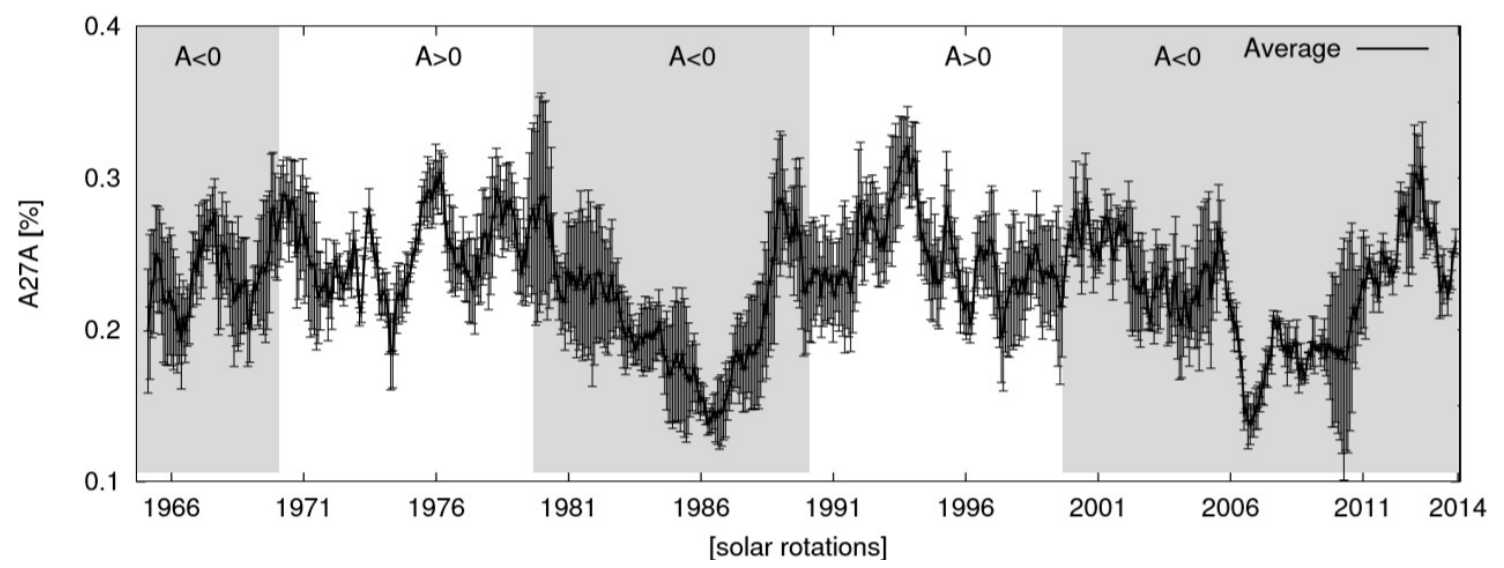

Fig. 1 Temporal changes of the average $A 27 A$ smoothed over 13 solar rotations for all considered NMs (Moscow, Kiel, Oulu, Deep River, Climax) for 1965-2014 during $A>0$ and $A<0$ polarity epochs; error bars are calculated as standard deviations for considered NMs.

However, these data are not homogenous and to study features of 3D anisotropy, (e.g. the 27 day variation of 3D anisotropy) we have to use results for Ar, Af and At obtained in different ways.

27-day variations of the GCR intensity [e.g., 21-26] and anisotropy [e.g., 27-28] have a sporadic character. Their amplitudes significantly increase and decrease averagely during 4-6 solar rotation periods. However, they are not completely random phenomena. Generally, there always exists some levels of amplitudes of the 27-day variations of the GCR intensity and anisotropy being above the background fluctuations. The 27-day variation of the GCR intensity in main is connected with heliolongitudinal asymmetry of solar wind and solar activity and their dependences on heliolatitudes, i.e. in creation of the 27-day variation of the GCR intensity contributes a fairly large part of the interplanetary space. At the same time, a convection diffusion mechanism of solar anisotropy [6-7] is determined by the local processes near the Earth orbit and requires less part of the heliosphere. Above all, especially in the solar activity minima epochs there seldom is observed the 27-day variation of the anisotropy apparently connecting with drift of cosmic rays in the sector structure of the HMF, while there is observed a feeble the 27-day variation in the GCR intensity. There can be observed a vice-versa circumstances, when a clear 27-day variation of the GCR intensity is observed, not following with the valuable 27-day variation of anisotropy at all. Unfortunately, we have not completely explained this phenomena, which indicates that problem of the 27-day variations of anisotropy 
and intensity is not fully understandable. Up to now we have not got available an universal mechanism of the 27-day variations of the anisotropy and intensity of GCR account for dynamical changes of the features of the sources in the solar atmosphere and heliosphere. So, up to now there still remains much necessity to find new properties of the 27-day variations of anisotropy and intensity of GCR. We think that among others, this problem is an important aim of this paper.

\section{27-day variation of the north-south anisotropy}

To study the 27-day variation of the north-south anisotropy we use two types of data: (1) daily At component calculated by GSM for NMs data (http://cr0.izmiran.ru/CosmicRayAnisotropy/) and (2) daily GG index obtained for MTs (http://www.stelab.nagoya-u.ac.jp/).

GG index [29] is the difference between intensities recorded in the geographically north (N2) south (S2) and east (E2)-viewing directional channels for 49 degrees inclination corresponding to median rigidity $60 \mathrm{GV}$ and calculated as follows: $\mathrm{GG}=(\mathrm{N} 2-\mathrm{S} 2)+(\mathrm{N} 2-\mathrm{E} 2)$.

This method for studying the north-south asymmetry has an advantage of using GCR intensity data from a single location, instead of comparing GCR intensities from north and south polar NMs. The GG index introduced according to the statement of Mori and Nagashima [29] should be free of noise in isotropic intensity caused by Forbush decreases, periodic variations, atmospheric temperature effects, and geomagnetic cut-offs. Although counting rate of GCR intensities in different directions (N2, S2, E2) could not precisely contain the same type of information, in spite GG is accepted as a good alternative index by worldwide cosmic ray community, e.g. Munakata et al. [30].

Recently, we analyzed [31] a behavior of the quasi-periodic changes of the GG index for 2007-2012 utilizing wavelet time-frequency method.
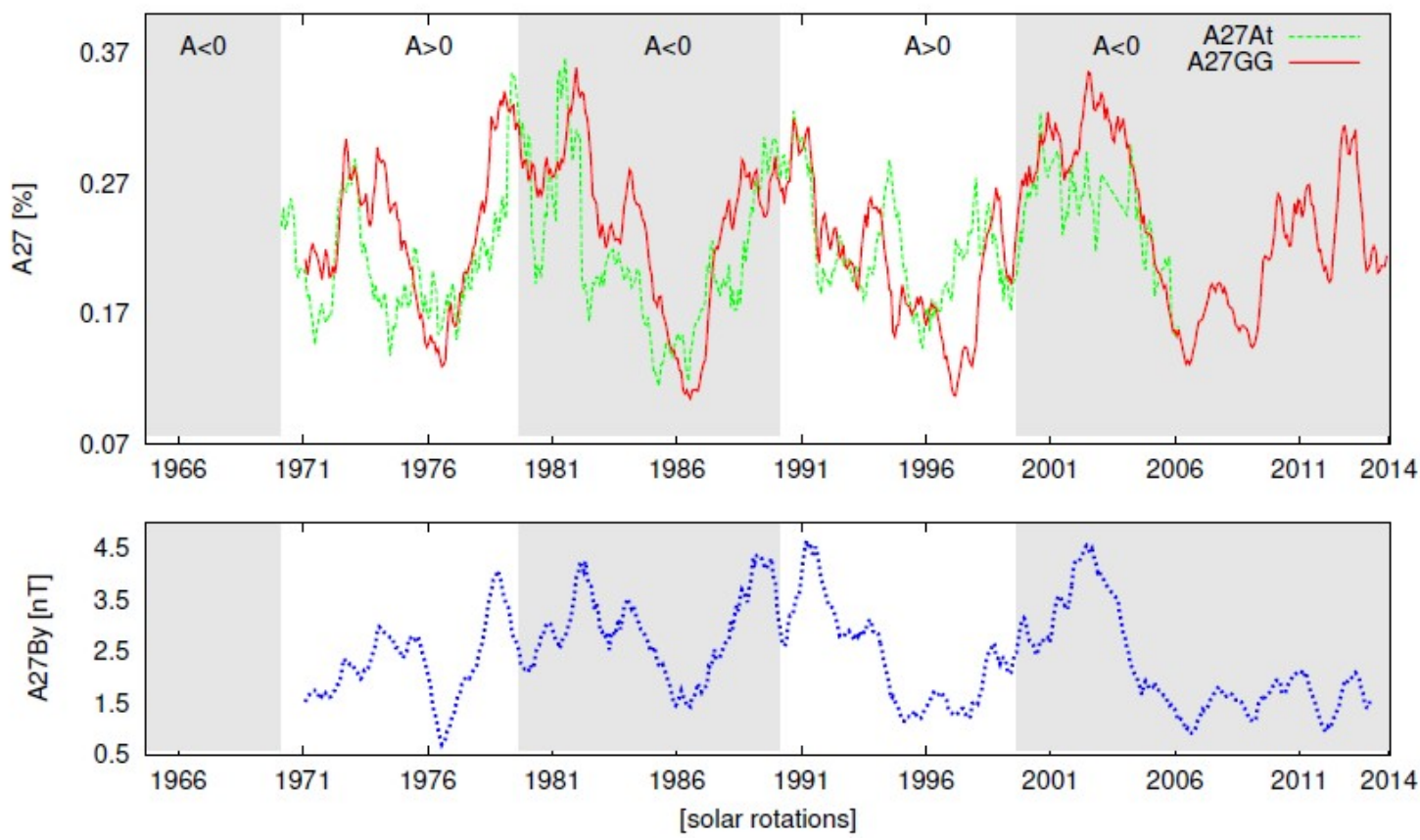

Fig. 2 Temporal changes of the amplitudes of the 27-day variation of the $G G$ index $(A 27 G G)$ and $A t$ component $(A 27 A t)$ of the 3D anisotropy (top) and $B y$ component of the HMF (A27By) (bottom) smoothed 
over 13 Sun's rotations during $A>0$ and $A<0$ polarity epochs. Values $A 27 G G$ and $A 27 B y$ are presented for 1971-2014, but $A 27 A t$ - for 1970-2006, data of $A t$ is available at the IZMIRAN website up to 2006.

\section{Long period changes of the amplitudes of the 27-day variations. 2D ecliptic plane anisotropy and north-south anisotropy}

To study long period changes of the amplitudes of the 27-day variation of the $2 \mathrm{D}$ ecliptic plane GCR anisotropy we calculate the amplitudes of the 2D 27-day variation of the anisotropy (A27A) [13] for Climax, Deep River, Kiel, Moscow, Oulu NMs data for 1965-2014.

Figure 1 shows the temporal changes of the average amplitude of the 27-day variation of the $2 \mathrm{D}$ GCR anisotropy in the ecliptic plane smoothed over 13 solar rotations for Climax, Deep River, Kiel, Moscow, Oulu NMs in the time interval 1965-2014; error bars are calculated as standard deviations for considered NMs. Due to smoothing over 13 solar rotations the error bars are reduced, but at the same time, we lost information about quasi periodic changes for time interval less than one year. Figure 1 demonstrates that the average amplitude of the 27-day variation of the GCR 2D anisotropy is larger in the minima periods of the positive $(A>0)$ polarity epochs than in the negative $(A<0)$ epochs, being in good agreement with [13-14]. In addition the time line of the amplitudes of the 27-day variation of the 2D GCR anisotropy shows a tendency of the 22-year variation and a feeble, but systematic decreasing near the periods of solar minimum for $\mathrm{A}>0$ polarity as well, indicating an existence of a weak 11-year solar cycle modulation.

The ecliptic 2D anisotropy components reflect significantly the drift pattern of cosmic rays flow and consequently the polarity dependence is apparent in the 27-day variation of the 2D GCR anisotropy.

To study the nature of the north-south asymmetry we calculate the amplitudes of the 27day variations of the GG index (A27GG), At (A27At) and By components (A27By) using harmonic analysis method for 1971-2014. The results we present in Fig. 2 Figure 2 demonstrates the temporal changes of the A27GG and A27At (top), and A27By (bottom) for 1970-2014. A behavior of the amplitudes of the 27-day variations of the GG index and At component is similar and highly positively correlated with the 27-day variation of the By component; all are alternating according to the 11-year solar cycle.

Contrary to the GCR anisotropy in the ecliptic plane, the north-south component has not got clear magnetic polarity dependence, e.g. Munakata et al. [30], so its 27-day recurrence also should not be dependent on the magnetic polarity, which we observe by experimental data.

\section{Discussion and conclusions}

We study long term changes of the amplitudes of the 27-day variation of the 3D GCR anisotropy for 1965-2014. We recognize a tendency of the 22-year changes of the amplitudes of the 27-day variation of the 2D anisotropy connected with the solar magnetic cycle and a feeble 11-year solar cycle modulation. We demonstrate that the amplitudes of the 27-day variation of the north-south component of the anisotropy vary in accordance with 11 year solar cycle, while there is hardly possible to show any dependence on the global solar magnetic field polarity. The 27-day recurrences of the GG index and At component are in a high positive correlation, and both are highly correlated with By component of the HMF. The observed properties of the 
radial Ar, azimuthal Af, normal At components and GG index demonstrate that quasi-periodic behaviors of the ecliptic 2D and north-south components of the 3D GCR anisotropy are governed by different mechanisms in the ecliptic plane and in the north-south direction.

\section{Acknowledgements}

We thank providers of data used in this study, especially OMNI database, IZMIRAN group and PIs of Oulu, Moscow, Kiel, Deep River, Climax NMs and Nagoya MTs.

\section{References}

[1] K. Kudela and I. Sabbah, Science China Technological Sciences 59 (2016) 547

[2] P. Chowdhury, K. Kudela, Y. J. Moon, Solar Phys. 291 (2016) 581

[3] G. Bazilevskaya, A.M. Broomhall, Y. Elsworth, V. M. Nakariakov, Sp. Sci. Rev. 186 (2014) 359

[4] K. Munakata, Ch. Kato, T. Kuwabara, J. Bieber, P. Evenson, M. Rockenbach, et al., Earth, Planets and Space 66 (2014) 151

[5] H. S. Ahluwalia and A.J. Dessler, Planet. Space Sci. 9 (1962) 195

[6] E. N. Parker, Planet. Space Sci. 12 (1964) 735

[7] G. F. Krymsky, Geomagnetism and Aeronomy 4 (1964) 763

[8] J. Chen and J. W. Bieber, Astrophys. J. 405 (1993) 375

[9] D. B. Swinson, J. Geophys. Res. 74 (1969) 5591

[10] H. S. Ahluwalia et al., J. Geophys. Res. Space Phys. 120 (2015) 8229- 8246

[11] I. Sabbah, J. Geophys. Res. 118 (2013) 4739

[12] S. Y. Oh, Y. Yi, J. Bieber, Solar Phys. 262 (2010) 199

[13] M. V. Alania, A. Gil, K. Iskra, R. Modzelewska, Proc. 29th ICRC SH3.4 (2005) 215

[14] M. V. Alania, A. Gil and R. Modzelewska, Adv. Space Res. 41 (2008) 280

[15] A. J. Owens, S. P. Duggal, M. A. Pomerantz, M. F. Tolba, Astrophys. J. 236 (1980) 1012

[16] D. B. Swinson and S.I. Yasue, Proc. 22nd ICRC SH (1991) 481

[17] D. B. Swinson and S.I. Yasue, J. Geophys. Res. 92 (1992) 19149

[18] D. B. Swinson, S.I. Yasue and Z. Fujii, Proc. 23rd ICRC SH (1993) 671

[19] D. B. Swinson and Z. Fujii, Proc. 24th ICRC SH (1995) 576

[20] A. Gil, R. Modzelewska, M. V. Alania, Advances in Space Research 50 (2012) 712

[21] I. G. Richardson, H. V. Cane, G. Wibberenz, J. Geophys. Res. 104 (1999) 12549

[22] P. Dunzlaff, B. Heber, A. Kopp, O. Rother, R. Müller-Mellin, A. Klassen et al., Ann. Geophys. 26 (2008) 3127

[23] X. Guo and V. Florinski, Journal of Geophysical Research: Space Physics 119 (2014) 2411

[24] X. Guo and V. Florinski, 2016, Astrophys. J. 826 (2016) 65

[25] A. Gil and K. Mursula, Astronomy and Astrophysics 599 (2017) A112

[26] A. Kopp et al., Astrophys. J. 837 (2017) 37

[27] R. Modzelewska and M. V. Alania, Advances in Space Research 50 (2012) 716

[28] H. Mavromichalaki, Ch. Papageorgiou, M. Gerontidou, Astrophysics and Space Science 361 (2016) 69

[29] S. Mori and K. Nagashima, Planetary and Space Science 27 (1979) 39

[30] K. Munakata, M. Kozai, C. Kato, J. Kota, Astrophys. J. 791 (2014) 22 
[31] R. Modzelewska and M.V. Alania, Journal of Physics: Conference Series 632 (2015) 1742 